\title{
Failure of visual prototype learning in the pigeon
}

\author{
SHIGERU WATANABE \\ Keio University, Tokyo, Japan
}

\begin{abstract}
Pigeons did not show generalization gradients along a distortion level after single stimulus training with dot patterns produced on a computer-controlled CRT. After discrimination training between a triangle made of six dots versus patterns made up of six random dots, the birds showed orderly generalization gradients as function of degree of distortion of the original triangle. When they were trained with distorted exemplars of the prototype triangle, they responded more often to triangles at this level of distortion than to the prototype triangle. This observation suggests a lack of abstraction of the prototype from its distorted samples. Such failure of prototype learning was also obtained after training with a different $S-$ (a square made up of six dots) or with a different $\mathrm{S}+$ (horizontally arranged dots).
\end{abstract}

Modern experimental research on animal perceptual concept learning began with Herrnstein and Loveland (1964), who demonstrated that pigeons could discriminate between photographs showing the presence or absence of human beings. Since then, there have been many studies that have reported "concept discrimination" in pigeons. This has included the concepts of a human (Herrnstein et al., 1976; Malott \& Siddall, 1972; Siegel \& Honig, 1970), a pigeon (Poole \& Lander, 1971), of a fish (Herrnstein \& de Villiers, 1980), an oak leaf (Cerella, 1979), a man-made object (Lubow, 1974), and so on. In addition to discriminations based on such "natural concepts," birds have successfully discriminated complex visual shapes (Ferraro \& Grishman, 1972; Hrycenko \& Harwood, 1980; Labiale, 1979), symmetry (Delius \& Habers, 1978), and the letter " $A$ " (Morgan, Fitch, Holman, \& Lea, 1976). These studies suggest that birds may be able to form visual concepts comparable to those of humans. For example, Blough (1982) applied cluster analysis to pigeons' confusions of alphabetic letters, and showed a similarity in how pigeons and humans classified the letters.

However, other work suggests that there are important species differences in the visual concepts of humans and other animals. For example, although humans can more easily perceive a partially occluded triangle as a "triangle" than as a partial triangle, the reverse has been observed to be true in pigeons (Cerella, 1980). Cerella (1980) also reported that in addition to being able to learn a visual concept of "Charlie Brown," pigeons can also respond to a random mixture of his legs, head, and trunk as if they made an intact image of Charlie Brown. A failure to make object-line-drawing transfers (Cabe, 1976) and a lack in the capacity to integrate transformations of perspective (Cerella, 1977) have also been found in the

The author extends his thanks to $S$. Chase and $E$. Heinneman for their helpful comments and corrections on an earlier draft of this manuscript. The author's mailing address is Department of Psychology, Keio University, Mita 2-15-45, Minato-Ku, Tokyo, Japan. pigeon. Thus, although both humans and pigeons can identify visual patterns, their processing strategies seem to be different.

In human experimental psychology, perceptual concept learning has been investigated primarily from a cognitive perspective. Early experimental work emphasized economy of data reduction in human memory (e.g., Attneave, 1957; Attneave \& Arnoult, 1956). Posner and Keele (1968), who showed that after learning a set of distorted patterns, human subjects could easily classify the prototype of the distorted patterns, proposed a prototype distance model to explain these interesting results. Other models, such as the attribute-frequency model (Neumann, $1974,1977)$ and the category-density model (Fried \& Holyoak, 1984), also predict abstraction of the prototype from its distorted derivatives.

Therefore, no information has been available about animals' capacities to abstract the prototype from distorted samples. The purpose of the present experiments was to compare prototype learning in humans and pigeons.

\section{EXPERIMENT 1 SINGLE-STIMULUS TRAINING}

Pigeons were trained to peck a pattern of dots that formed a triangle. The pattern was a triangle formed by six dots (the prototype) for one group and a distorted version of that triangle for another group. There was a short blackout period between trials but no other $S-$. After training, the birds were given a generalization test with triangle patterns at different levels of distortion.

\section{Method}

Subjects. Eight experimentally naive pigeons (Columba livia) were used. They were maintained at about $80 \%$ of their free-feeding weights.

Stimuli. The training pattern was a triangle pattern formed by six dots. Each dot occupied 1 pixel. The pattern was displayed on a computer-controlled CRT screen. The height of the triangle was $1.4 \mathrm{~cm}$ (40 pixels); the width of the base $2.5 \mathrm{~cm}$ (80 pixels). Distorted triangles were made as follows. The position of each dot in 
the original prototype triangle was moved to one of the surrounding four pixels (1 pixel up or down and 1 pixel left or right) at Distortion Level 1, and 4 pixels on the next surrounding area (2 pixels up or down and 2 pixels left or right) at Distortion Level 2, and so on. These distortions were determined at each presentation through the use of the Applesoft random generator function. This procedure generated 4,096 possible distorted patterns for each distortion level. Examples of the stimuli are shown in Figure 1.

Apparatus. The experimental chamber was an operant chamber for pigeons $(30 \times 30 \times 30 \mathrm{~cm})$. The stimuli were presented on a computer-controlled CRT screen $(10 \times 7.5 \mathrm{~cm}$; Victor CX-64) positioned $1 \mathrm{~cm}$ behind a transparent rectangular pecking key $(9 \times 6 \mathrm{~cm})$. The distance from the key to the floor of the chamber was $19 \mathrm{~cm}$. The grain hopper for reinforcement was mounted below the key. Continuous noise from a fan was present during the experiment. The experiment was controlled by a microcomputer system (Apple II) in another room.

Procedure. All subjects were shaped to peck a key by an autoshaping procedure. Then they were divided into two groups of 4 each. The prototype group was trained with the original triangle pattern, and the distortion group was trained with distorted triangles at Distortion Level 2. One daily training session consisted of 40 presentations of the training stimulus, each lasting $30 \mathrm{sec}$ and followed by a 3-sec blackout period. Responding for the training stimulus was reinforced on a variable-interval (VI) 20-sec schedule. Reinforcement was 3-sec access to the grain hopper. This training procedure continued for 40 sessions and was followed by a generalization test. During the test, triangle patterns at six distortion levels (Level 0 to Level 5) were presented six times in accordance with a $6 \times 6$ Latin square. Presentation periods were $30 \mathrm{sec}$ long and were separated from each other by a 3-sec blackout period. No reinforcement was available during the test. The generalization test was repeated again after a single retraining session.

\section{Results and Discussion}

Figure 2 presents results of the generalization test for the prototype group. Rate of response for each stimulus is expressed as a percentage of the rate for the original stimulus (Level 0 ). None of the birds showed systematic generalization along the dimension of distortion level. Results of the generalization test for the distortion group are presented in Figure 3. Although Bird 331 tended to respond more in the presence of the less distorted patterns, none of the other birds showed orderly generalization gradients. The present results indicate that singlestimulus training is not sufficient to establish dimensional stimulus control along distortion level.

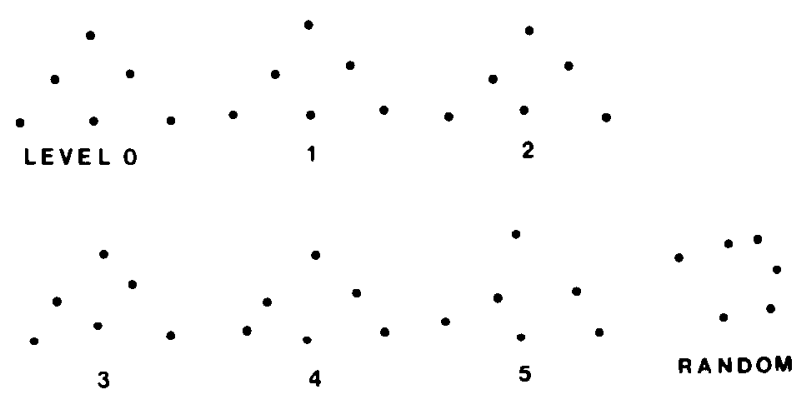

Figure 1. Examples of distortions of the prototype triangle (Level 0) and random dots. (The size of the dots is not actual.)

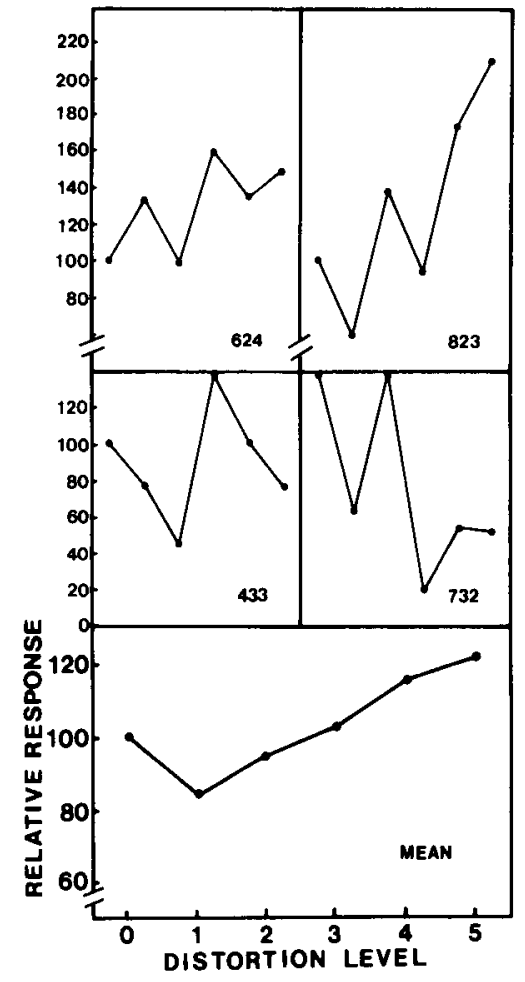

Figure 2. Results of the generalization test after single-stimulus training with a prototype triangle. The upper four panels present individual results (average of two tests). The number of responses for each stimulus is expressed as a percentage of the responses to the training stimulus (Level 0 ).

\section{EXPERIMENT 2 DISCRIMINATION TRAINING}

In this experiment, pigeons were required to discriminate between a pattern of dots that formed a triangle and patterns of random dots. For one group, $\mathrm{S}+$ was the prototype triangle; for another group, $\mathrm{S}+$ was triangles at Distortion Level 2.

\section{Method}

Subjects. The subjects were 8 experimentally naive pigeons. They were maintained at $80 \%$ of their free-feeding weights.

Stimuli. The original triangle and its distorted versions were generated by the method described for Experiment 1. The six random dots were displayed on a $95 \times 56$ pixel area, and one dot $0 c-$ cupied 1 pixel. Examples of the random-dot patterns are shown in Figure 1. For each presentation, the dot positions were determined by the Applesoft random generator function.

Apparatus. The apparatus was identical to that used in Experiment 1.

Procedure. All subjects were shaped to peck the key. Then they were divided into two groups of 4 . After a few days of peck training on a VI schedule, discriminative training was begun. Each daily training session consisted of 20 presentations of $\mathrm{S}+$ and 20 presentations of S-. Each stimulus-presentation period lasted $30 \mathrm{sec}$ and was followed by a 3-sec blackout period. For the prototype group, $\mathrm{S}+$ and $\mathrm{S}-$ were the original triangle and random patterns; for the 


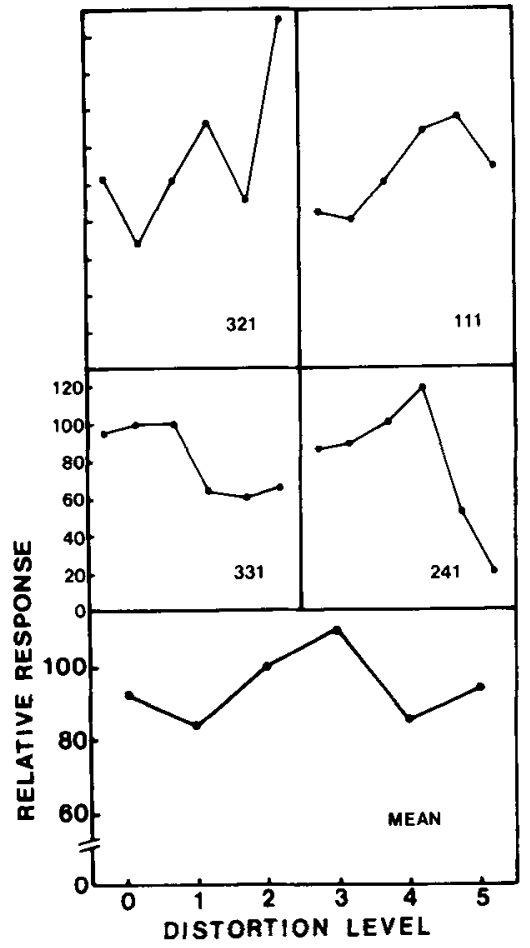

Figure 3. Results of the generalization test after single-stimulus training with distorted triangles (Level 2). The number of responses for each stimulus is expressed as a percentage of the responses to the training stimulus (Level 2).

distortion group, they were the Level 2 distorted triangles and random-dot patterns. The random patterns and the distorted triangles were generated by the random function at each presentation.

Responding during the $S+$ period was reinforced on a VI 20 -sec schedule, and responding during the $S-$ period was extinguished on a mult VI-EXT schedule. S+ and S- periods were alternated randomly with the constraint that no more than two successive presentations be of the same stimulus. The training continued until the subjects showed above $90 \%$ correct responses, calculated by dividing the number of responses to $S+$ by the total number of responses on two successive sessions.

When each bird reached this criterion of discrimination, it was given generalization tests identical to those described in Experiment 1 .

\section{Results and Discussion}

The number of sessions required for each bird to attain the criterion of discrimination was $18,35,37$, and 37 in the prototype group and $27,34,82$, and 93 in the distortion group. Discrimination between the distorted triangles and random dots seems to be a difficult task, at least for some pigeons.

Figure 4 presents the results of the generalization test for the prototype group. The birds showed orderly generalization gradients, with peaks at the original S+ stimulus, except for 1 bird (333), whose maximum was at Distortion Level 1 . Thus, the pigeons showed stimulus control along the dimension of distortion level after discriminative training.
Figure 5 shows the generalization gradients of the birds in the distortion group. The gradients are orderly, with the peak of S+ responding at Distortion Level 2 for 3 of the 4 birds and at Distortion Level 3 for the remaining bird. The absence of a peak at the prototype indicates that the birds learned during their discriminative training to peck at a triangle at Distortion Level 2. They did not abstract the prototype from its distorted samples. These results do not support an averaging model of prototype abstraction. In the present experiment, at each distortion level, each dot had an equal probability of appearing in one of four possible positions. These positions were equidistant (left or right; above or below) from the dot on the prototype triangle. Their average position was the prototype position. Under the distortion condition, a dot never appeared in this position. In Posner's experiments, exemplars could have points in the prototype position.

\section{EXPERIMENT 3}

The results of Experiment 2 clearly demonstrated a failure of prototype learning in the pigeon. However, since the failure might be due to the particular $\mathrm{S}+$ and $\mathrm{S}-$ stimuli that were used, different $S+$ and $S-$ stimuli were employed in Experiment 3.

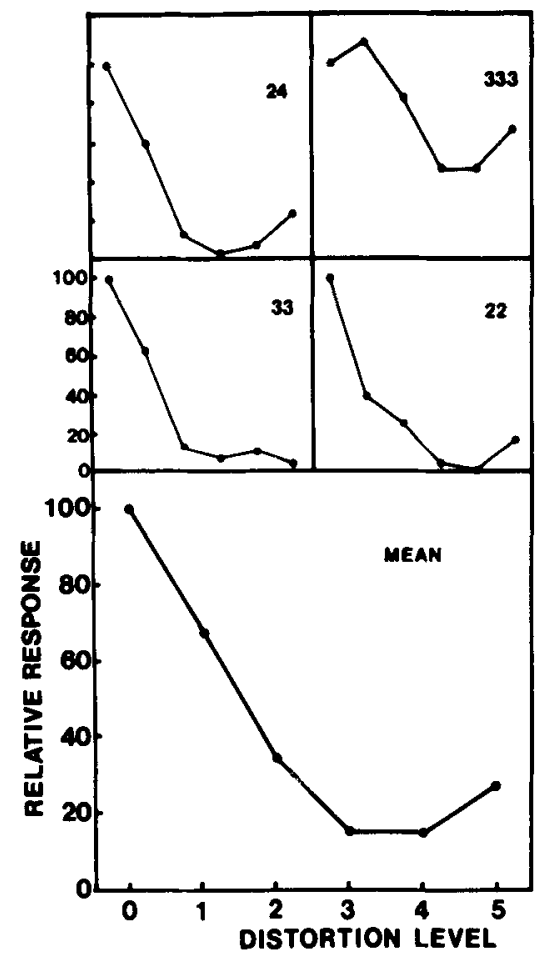

Figure 4. Generalization test after discrimination training between the prototype triangle and random dots. The number of responses for each stimulus is expressed as a percentage of the responses to the $\mathbf{S}+$ (the prototype). 


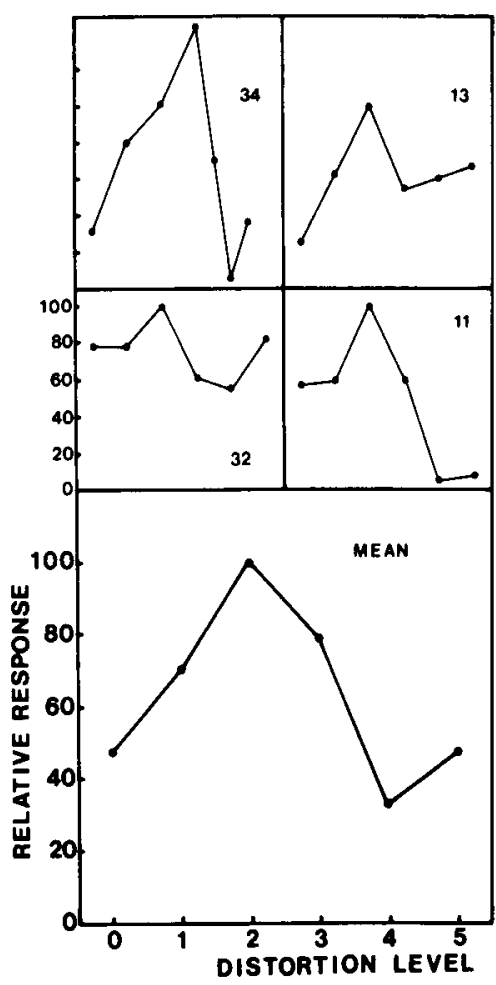

Figure 5. Results of the generalization test after discrimination training between distorted triangles (Level 2) and random-dot patterns. The number of responses for each stimulus is expressed as a percentage of responses to the S+ (Level 2).

\section{Method}

Subjects. Eight experimentally naive pigeons were used. They were maintained at $80 \%$ of their free-feeding weights.

Stimuli. Two new stimuli were introduced in this experiment. One was $2.5 \times 2.5 \mathrm{~cm}$ square made up of six dots. There were three dots on the vertical line and two on the horizontal line. The second new pattern consisted of six dots arranged horizontally in a 12.6cm-long line. The horizontally arranged dots were distorted using the same method used to produce the distorted triangles in the previous experiments.

Apparatus. The apparatus was identical to that used in Experiments 1 and 2.

Procedure. The subjects were shaped to peck the key, and then divided into two groups of 4 . The square-S- group received triangle versus square discrimination training. The $S+$ was the same Level 2 distorted triangles used in Experiment 2, but the $S-$ was a square pattern rather than a random-dot one. The horizontal-S+ group received horizontally arranged dot versus random dot discrimination training. Horizontally arranged dot patterns at Distortion Level 2 were the S+, and sets of six randomly placed dots, dots like those used in Experiment 2, were the S- . Other training procedures were identical to those used previously. After the subjects reached the criterion of discrimination, the square-S- group received the same generalization tests that had been given in the previous experiments. The horizontal S+ group received generalization tests in which six horizontally arranged dot patterns with Level 0 to Level 5 distortions were presented six times each in accordance with a $6 \times 6$ Latin square. In all other respects, the test procedures were the same as those used in the previous experiments.

\section{Results and Discussion}

The birds in the square-S- group required 10, 10, 15, and 26 sessions to reach criterion. Those in the
horizontal-S+ group required $9,19,29$, and 36 sessions. Thus, for pigeons, these discriminations seem to be easier than the distorted triangle versus random dot discrimination of Experiment 2.

Figure 6 shows the results of the generalization tests given in the square-S- group. Clearly, none of the pigeons showed prototype learning after this training. Furthermore, every subject had a peak at Level 3 rather than at Level 2 or Level 0 . A peak shift was observed. The sides of the square pattern ( $\mathrm{S}-$ ) consisted of dots that were aligned along vertical and horizontal lines, but the dots that made up the distorted triangles ( $\mathrm{S}+$ ) did not lie along straight lines. Such features of the discriminative stimulus might result in a preference for more distorted patterns.

Results of the generalization test in the horizontal-S+ group are presented in Figure 7. All subjects show a peak at the original S+. Although they responded more often to the less distorted patterns, no bird had a peak at the prototype, the pattern of six horizontal dots. Thus, the birds did not show prototype learning with the horizontally arranged dots.

\section{GENERAL DISCUSSION}

When dot patterns displayed on a CRT screen were used as stimuli, discrimination training was necessary to produce stimulus control by the dimension of distortion level. Thus, acquisition of such stimulus control seems to be different from that for color stimuli (Guttman \& Kalish, 1956). When discriminative training was in-

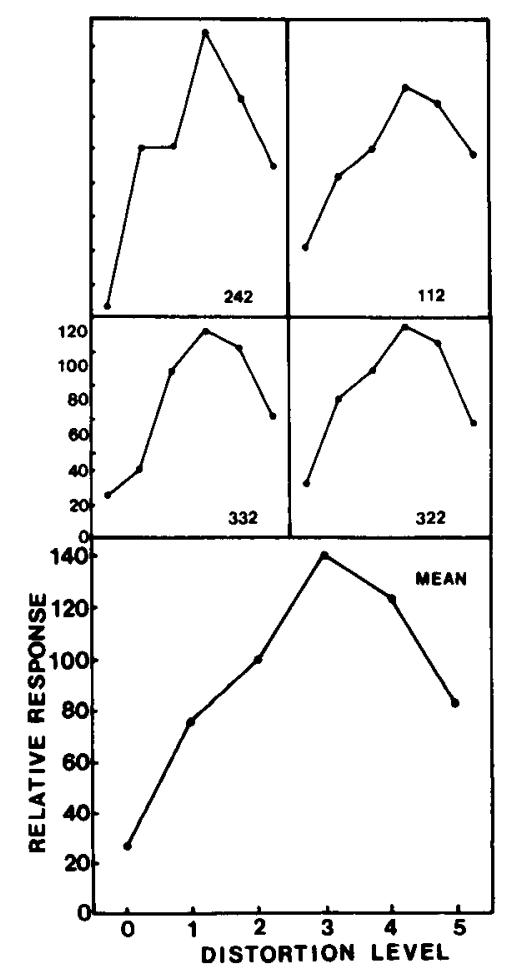

Figure 6. Results of the generalization test after discrimination training with the square $S$-. The number of responses for each stimulus is expressed as a percentage of the responses to the $S+$ (Level 2). 


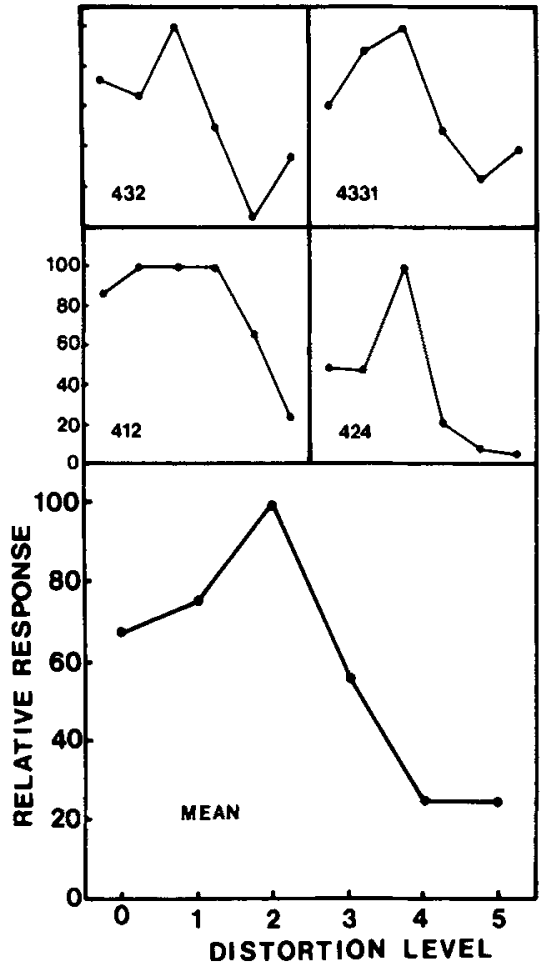

Figure 7. Results of the generalization test after discrimination training with horizontally arranged dots as the $S+$. The responses to each stimulus are expressed as a percentage of the responses to the $\mathbf{S}+$ (the horizontal dots distorted at Level 2 ).

troduced, dimensional control by distortion level was clearly observed. Following discrimination training, the degree of "distortion" of dot patterns constitutes a dimension along which a generalization decrement can be observed. However, it must be pointed out that the birds required many training sessions to learn these discriminations, especially when the training pattern was a distorted one.

The most important finding of the present experiments is the failure to find prototype learning in Experiments 2 and 3. When human subjects were trained to discriminate between a distorted triangle and random dots in a similar situation and asked to rate the similarity of the test patterns to the training stimulus, they gave the highest similarity score to the prototype (Level 0 stimulus) and not to the training stimulus (Level 2 stimulus) (personal observation). Although there are many problems associated with a direct comparison between human rating scores and pigeons' generalization gradients, these results suggest differences between human and pigeon prototype learning.

Experiment 3 showed that the pigeon's inability to conceptualize abstract prototypes is not specific to one particular set of stimuli. There have been some experiments involving color naming by pigeons (e.g., Zentall \& Edwards, 1984), but the results do not support the averag- ing model, since the training stimulus was not centered around one prototype color but, rather, a color that was close to some specific wavelength.

What factors cause the failure of prototype learning in pigeons? Interestingly, some human subjects described the training stimulus (distorted pattern) as a distorted triangle rather than simply as a triangle, but they gave the highest similarity score to the prototype instead of to the training stimulus (personal observation). Observations such as these suggest that verbal coding must play at least a minor role in the way humans approach the task. Pigeons lack such codes. It is still an open question as to whether the pigeon could abstract the prototype from its distorted samples if some kind of coding were available to them.

\section{REFERENCES}

Attneave, F. (1957). Physical determinants of the judged complexity of shapes. Journal of Experimental Psychology, 53, 221-227.

Attneave, F., Arnoult, M. D. (1956). The quantitative study of shape and pattern perception. Psychological Bulletin, 53, 452-471.

BLouch, S. (1982). Pigeon perception of letters of the alphabet. Science, 218, 397-398.

CABE, P. A. (1976). Transfer of discrimination from solid objects to pictures by pigeons: A test of theoretical models of pictorial perception. Perception \& Psychophysics, 19, 545-550.

Cerella, J. (1977). Absence of perspective processing in the pigeon. Pattern Recognition, 9, 65-68.

Cerella, J. (1979). Visual classes and natural categories in the pigeon. Human Perception \& Performance, 5, 68-77.

Cerella, J. (1980). The pigeon's analysis of pictures. Pattern Recognition, 12, 1-6.

Deuuus, J. D., \& Habers, G. (1978). Symmetry: Can pigeons conceptualize it? Behavioral Biology, 22, 336-342.

Ferraro, D. P., \& Gusham, M. G. (1972). Discrimination and generalization of complex visual shape variations in pigeons. Perceptual \& Motor Skills, 35, 915-927.

FrIED, L. S., HoLYOAK, K. J. (1984). Induction of category distributions: A framework for classification learning. Joumal of Experimental Psychology: Learning, Memory, \& Cognition, 10, 234-257.

Gutrman, N., KaLISH, H. I. (1956). Discriminability and stimulus generalization. Joumal of Experimental Psychology, 51, 79-88.

Herrnstein, R. J., \& DE Vililers, P. A. (1980). Fish as a natural category for people and pigeons. In G. H. Bower (Ed.), The psychology of learning and memory. New York: Academic Press.

Herrnstein, R. J., \& Loveland, D. H. (1964). Complex visual concept in the pigeon. Science, 146, 549-551.

Herrnstein, R. J., Loveland, D. H., \& Cabe, C. (1976). Natural concepts in pigeons. Joumal of Experimental Psychology: Animal Behavior Processes, 2, 285-302.

HrYCENKo, O., HARWOOD, D. W. (1980). Judgment of shape similarity in the barbary dove (Stereptopella risoria). Animal Behaviour, 28, 586-592.

LABIALE, G. (1979). La discrimination visuelle de formes complexes chez le merle, Turdus merula (L). Behavioural Processes, 4, 173-177.

LUBOw, R. E. (1974). Higher order concept formation in the pigeon. Journal of the Experimental Analysis of Behavior, 21, 475-483.

Malott, R. W., \& Siddall, J. W. (1972). Acquisition of the people concept in pigeons. Psychological Report, 31, 3-13.

Morgan, M. J., Fitch, M. D., Holman, J. G., \& Lea, S. E. G. (1976). Pigeons learn the concept of an 'A.' Perception, 5, 57-66.

NeumanN, P. G. (1974). An attribute frequency model for the abstraction of prototypes. Memory \& Cognition, 2, 241-248.

NeumanN, P. G. (1977). Visual prototype formation with discontinu- 
ous representation of dimensions of variability. Memory \& Cognition, 5, 187-197.

Poole, J., \& LANDER, D. G. (1971). The pigeon's concept of pigeon. Psychonomic Science, 25, 157-158.

Posner, M. I., \& KeEle, S. W. (1968). On the genesis of abstract ideas. Journal of Experimental Psychology, 77, 353-363.

SIEGEL, R. K., \& HoNIG, W. K. (1970). Pigeon concept formation: Suc- cessive and simultaneous acquisition. Journal of the Experimental Analysis of Behavior, 13, 385-390.

Zentall, T. R., \& Edwards, C. A. (1984). Categorical color coding by pigeons. Animal Learning \& Behavior, 12, 249-255.

(Manuscript received August 6, 1987;

revision accepted for publication November 23, 1987.) 